\title{
Characterization of CZT detectors for the ASIM mission
}

\author{
Budtz-Jørgensen, Carl; Kuvvetli, Irfan; Skogseide, Y; Ullaland, K; Ostgaard, N
}

Published in:

IEEE Nuclear Science Symposium Conference Record, 2008. NSS '08.

Link to article, DOI:

10.1109/NSSMIC.2008.4775134

Publication date:

2008

Document Version

Publisher's PDF, also known as Version of record

Link back to DTU Orbit

Citation (APA):

Budtz-Jørgensen, C., Kuvvetli, I., Skogseide, Y., Ullaland, K., \& Ostgaard, N. (2008). Characterization of CZT detectors for the ASIM mission. In IEEE Nuclear Science Symposium Conference Record, 2008. NSS '08. (pp. 106-110). IEEE. https://doi.org/10.1109/NSSMIC.2008.4775134

\section{General rights}

Copyright and moral rights for the publications made accessible in the public portal are retained by the authors and/or other copyright owners and it is a condition of accessing publications that users recognise and abide by the legal requirements associated with these rights.

- Users may download and print one copy of any publication from the public portal for the purpose of private study or research.

- You may not further distribute the material or use it for any profit-making activity or commercial gain

- You may freely distribute the URL identifying the publication in the public portal 


\title{
Characterization of CZT Detectors for the ASIM Mission
}

\author{
C. Budtz-Jørgensen, I Kuvvetli Member, IEEE, Y. Skogseide, K. Ullaland, N. Ostgaard
}

\begin{abstract}
The National Space Institute, Technical University of Denmark is responsible for the selection and characterization of the CZT detector crystals for the $X$ and gamma -ray instrument MXGS onboard ESA's Atmospheric Space Interaction Monitor (ASIM) mission. The Modular X- and Gamma ray Sensor (MXGS) shall, situated on the International Space Station, detect Terrestrial Gamma ray Flashes (TGF) generated in the Earth atmosphere. TGFs are believed to be produced in connection with thunderstorms and ASIM will therefore also be equipped with optical instruments which trigger on lightening.

MXGS is equipped with a sensor plane consisting of 64 detector modules each having $4 \mathrm{CZT}$ crystals with dimensions of $20 \mathrm{~mm} \times 20 \mathrm{~mm} \times 5 \mathrm{~mm}$. The crystals are divided in pixels with a pitch of $2.5 \mathrm{~mm}$. The MXGS employs coded mask imaging with which the direction of the TGF with respect to Nadir can be determined. The MXGS spectral performance is important since the details of the TGF energy spectrum can be used to determine the atmospheric generation altitude of the TGFs.

Redlen has recently delivered the first CZT detector modules. Measurements at the University of Bergen demonstrate that the detectors exhibit the expected spectral performance; however it was also found that the detector modules showed unexplained pixel-to-pixel count rate variations. At The National Space Institute a series of measurements were initiated focusing on the pixel-to-pixel count rate variations. Collimated monochromatic $X$-ray beams were used to scan a number of Redlen pixel detectors. The main conclusion of these measurements is that the pixel-to-pixel count rate variations are likely related to the nonuniformity of the detector internal electric field. The measurements and their interpretation will be discussed in the following sections
\end{abstract}

\section{INTRODUCTION}

$\mathrm{T}^{\mathrm{s}}$ he Atmosphere-Space Interactions Monitor (ASIM) is proposed for the International Space Station externa facilities on the Columbus module, for the study of giant electrical discharges (lightning) in the high-altitude atmosphere above thunderstorms. The discharges are seen as optical-, X- and Gamma ray flashes in the stratosphere and mesosphere. The optical emissions are dubbed "red sprites", "blue jets", and "elves" or "Transient Luminous Events" (TLEs). The $\mathrm{X}$ - and Gamma ray emissions are dubbed "Terrestrial Gamma ray Flashes" (TGFs). The ASIM mission comprises therefore two main scientific instruments: The Miniature Multi-spectral Imaging Array (MMIA) composed of

\footnotetext{
Manuscript received November 7, 2008.

C. Budtz-Jørgensen and I. Kuvvetli are with the National Space Institute Technical University of Denmark (e-mail: carl@space.dtu.dk, e-mail irfan@space.dtu.dk).

Y. Skogseide, K. Ullaland and N. Ostgaard are with the Bergen University, Norway.
}

6 cameras and 6 photometers and the Modular X- and Gamma ray Sensor (MXGS) The ASIM payload is shown in Fig. 1.

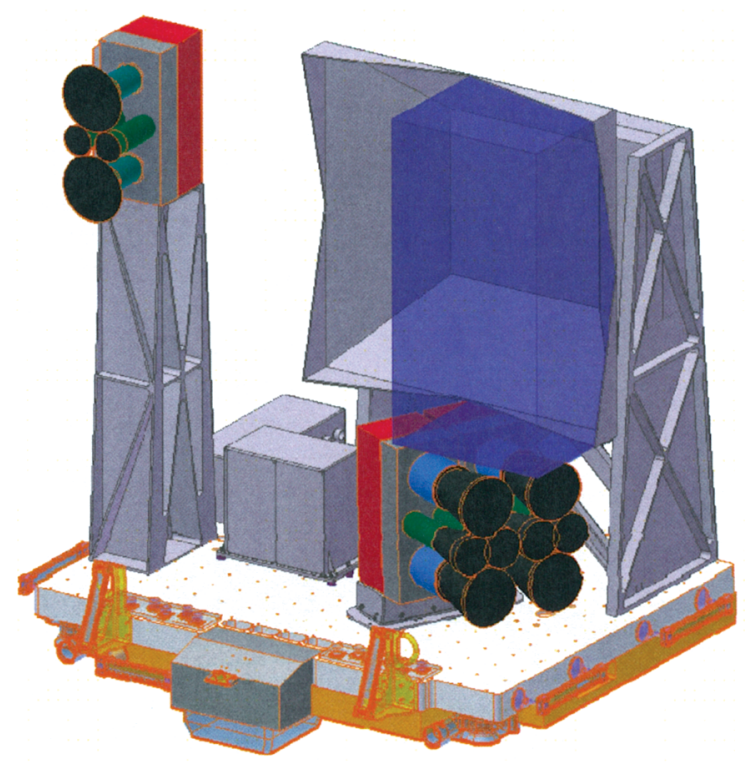

Fig. 1. The ASIM payload for the ISS Colombus external platform. The left tower supports the MMIA Nadir instrumentation. The right tower supports MXGS also pointing towards Nadir. The MMIA Limb optical instruments are placed below MXGS.

The ASIM mission will:

- Provide the most comprehensive survey of the occurrence of TLEs on a global scale and establish the relationship between TLEs and the thunderstorm source.

- Survey the occurrence of TGFs and establish the relationship between the TGFs, TLEs and other parameters characterizing atmospheric activity.

- Establish the relative importance of mechanisms proposed for the generation of sprites and jets.

- Quantify the effects of TLEs and TGFs on the upper atmosphere (stratosphere, mesosphere, lower thermosphere, and ionosphere).

- Quantify the interaction of TGF and lightninginduced electromagnetic radiation with the radiation belts. 
TABLE I

MXGS PERFORMANCE SPECIFICATIONS

\begin{tabular}{ll}
\hline ENERGY RANGE & $10-500 \mathrm{keV}$ \\
ENERGY RESOLUTION & $<10 \% @ 60 \mathrm{keV}$ \\
QUANT. EFF. & $>0.95 @ 100 \mathrm{keV}$ \\
ABSOLUTE TIME ACCURACY & $<100 \mu \mathrm{s}$ \\
BURST RATE CAPABILITY & $1000 \mathrm{cts} / \mathrm{ms}$ \\
SENSITIVE AREA & $1024 \mathrm{~cm}^{2}$ \\
TOTAL DETECTOR BACKGROUND & $\sim 2 \mathrm{cts} / \mathrm{ms}^{(10-500 \mathrm{keV})}$ \\
FIELD OF VIEW & $80^{\circ} 80^{\circ}$ \\
ANGULAR RESOLUTION & $2^{\circ}$. \\
\hline
\end{tabular}

\section{THE MOdUlaR X- AND GAMMA-RAY SENSOR (MXGS)}

The MXGS performance specifications are given in Table $I$. The detector plane consists of a $1024 \mathrm{~cm}^{2}$ array of CZT detector crystals. It is protected against the background radiation by a passive graded shield surrounding the detector housing. A Hopper shaped collimator defines the $80^{\circ} \times 80^{\circ}$ field of view for MXGS and shields the detector plane against the Cosmic X-ray Background. The Detector Front End Electronics (DFEE) is mounted in the housing below the detectors. The electronics contains also the High Voltage Power Supply (HVPS) and Low Voltage Power Supply (LVPS) as well as the MXGS Data Processing Unit (DPU).



Fig. 2. MXGS Detector Module.

The MXGS instrument DFEE design consists of 4 Detector Assembly Units (DAU), and each DAU consists of 16 Detector Modules (DM) and one Detector Assembly Board (DAB). The DAB holds the read-out electronics and the Readout Control Unit (RCU). The RCUs interface to the Data Processing Unit (DPU). The purpose of the DAU is to read out the events and transfer the data to the DPU.

The DM consists of two separable units, a CZT sensor and an ASIC. The sensor comprises four $20 \times 20 \times 5 \mathrm{~mm}^{3} \mathrm{CZT}$ detectors tiled together on a PCB. Each detector is pixelated into 64 pixels $(2.5 \mathrm{~mm}$ pixel pitch), making a $16 \times 16$-pixel array in total. The detector unit is stacked onto the ASIC unit via three connectors (see Fig. 2). The ASIC unit holds two XA 1.72 chips, each reading 128 pixels. The ASICs provide position and energy for each detected photon. The DM is operated through front end electronics and software developed at University of Bergen.

\section{A. CZT Material Selection}

At NSI we have conducted a campaign with the purpose of selecting the most suited CZT/CdTe detectors for MXGS. [1] The NSI test set-up comprises a 16 channel multi-parameter data acquisition system including 16 low-noise preamplifiers. A number of radioactive sources are available. A step motor facility can be loaded with these sources and fine scans with monochromatic beams can be performed for detailed analysis of the detector responses.

The tests were typically conducted on $10 \times 10 \times 5 \mathrm{~mm}$ $\mathrm{CZT} / \mathrm{CdTe}$ crystals with a pixel pitch of $2.5 \mathrm{~mm}$ similar to that of the DMs. As a result of these investigations we chose Redlen CZT material as the baseline for MXGS. Fig. 3 shows a photo of investigated Redlen detectors. The pixel gap is only 100 micrometers. Our investigations [1] have shown that a minimum pixel gap reduce the influence of pixel charge sharing and pixel gap charge losses.

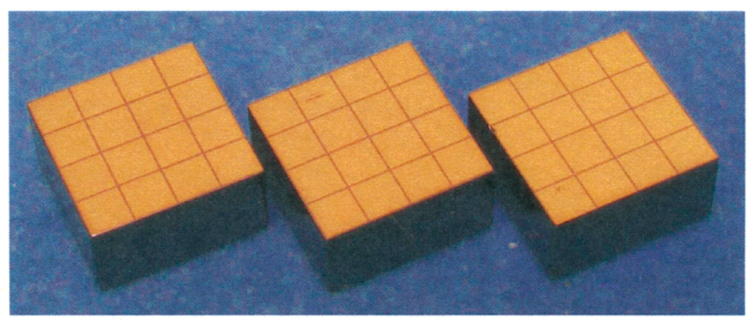

Fig. 3. Redlen CZT semples ( $10 \mathrm{~mm} \times 10 \mathrm{~mm} \times 5 \mathrm{~mm})$.

It is important that the Redlen material consistently showed very good charge collection properties with an electron mobility lifetime product $(\mu \tau)_{\mathrm{e}}>10^{-2} \mathrm{~cm}^{2} / \mathrm{V}$. This material is therefore well suited for detectors with fine energy resolution.
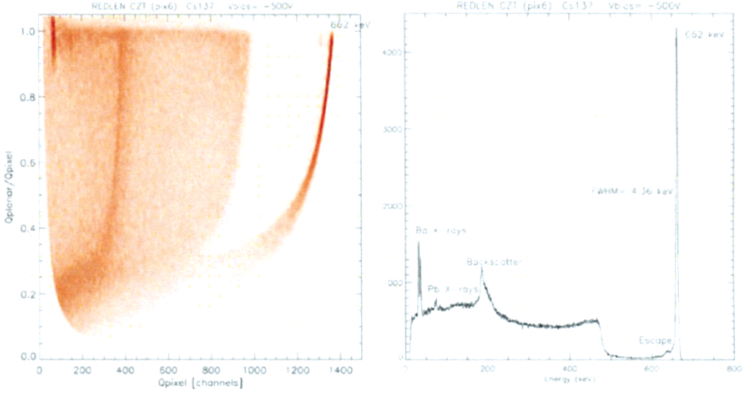

Fig. 4. Redlen pixel detector response to $137 \mathrm{Cs}$ photons. The left plot gives the bi-parametric distribution for the pixel signal versus the ratio between the cathode and the pixel signal. The right figure displays the resulting $137 \mathrm{Cs}$ spectrum after application of depth of interaction correction.

An example of the achievable quality is shown in Fig. 4. The presented results were obtained with a ${ }^{137} \mathrm{Cs}$ source. The left plot gives the bi-parametric distribution for the pixel 
signal versus the ratio between the common cathode and the pixel signal. The right figure displays the resulting ${ }^{137} \mathrm{Cs}$ spectrum after application of depth of interaction correction (DOI) as described in [2] and [3].

\section{MEASUREMENTS ON THE FIRST DMS}

Redlen has recently delivered the first set of CZT detector modules. Measurements at the University of Bergen on one of the DM connected to two XA 1.72 ASICs demonstrate that the detectors exhibit the expected spectral performance. This is illustrated in Fig 4 where the upper figure is a 2D image of $128{ }^{57}$ Co spectra from one of the ASICs. The middle plot shows a typical spectrum. The overall energy resolution measured for the $122 \mathrm{keV}$ line is $6.2 \%$ (FWHM) at $20^{\circ} \mathrm{C}$ and improved slightly to $5.5 \%$ (FWHM) at a detector temperature of $5^{\circ} \mathrm{C}$.
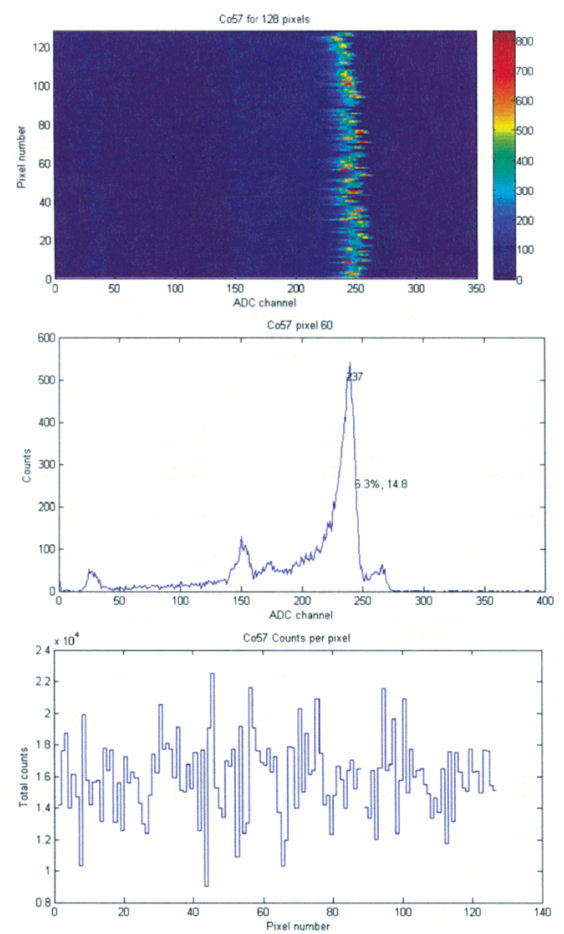

Fig. 5. DM measurements at UB. The upper figure is a $2 \mathrm{D}$ image of $128{ }^{57} \mathrm{Co}$ spectra from one of the ASICs. The middle plot shows a typical spectrum. Pixel-to-pixel count rate variations are shown in the lower plot.

However it was found that the detector modules showed large unexplained pixel-to-pixel count rate variations as it can be seen pixel count plot in the lower left part of Fig. 5. Here the pixel count distribution has a rms scatter of $20 \%$ with a max to min pixel count of more than a factor of 2 . This behavior is problematic since it might indicate that the DMs suffer from pixel varying inefficiencies and that the DM therefore has an overall efficiency which is lower than the predicted one.

\section{X-Y SCANS}

We decided to investigate the Redlen pixel detectors and the count rate variations in more detail performing X-Y scans with fine X-ray beams. The early detectors shown in. Fig. 3 showed a similar pixel to pixel variation. We decided to start with these detectors since they were easier to interface to the NSI data acquisition set-up than the DMs. The NSI facility comprises also a 2D step motor equipment which allows to scan (position accuracy $<5$ micrometers) the detectors with collimated X-ray beams
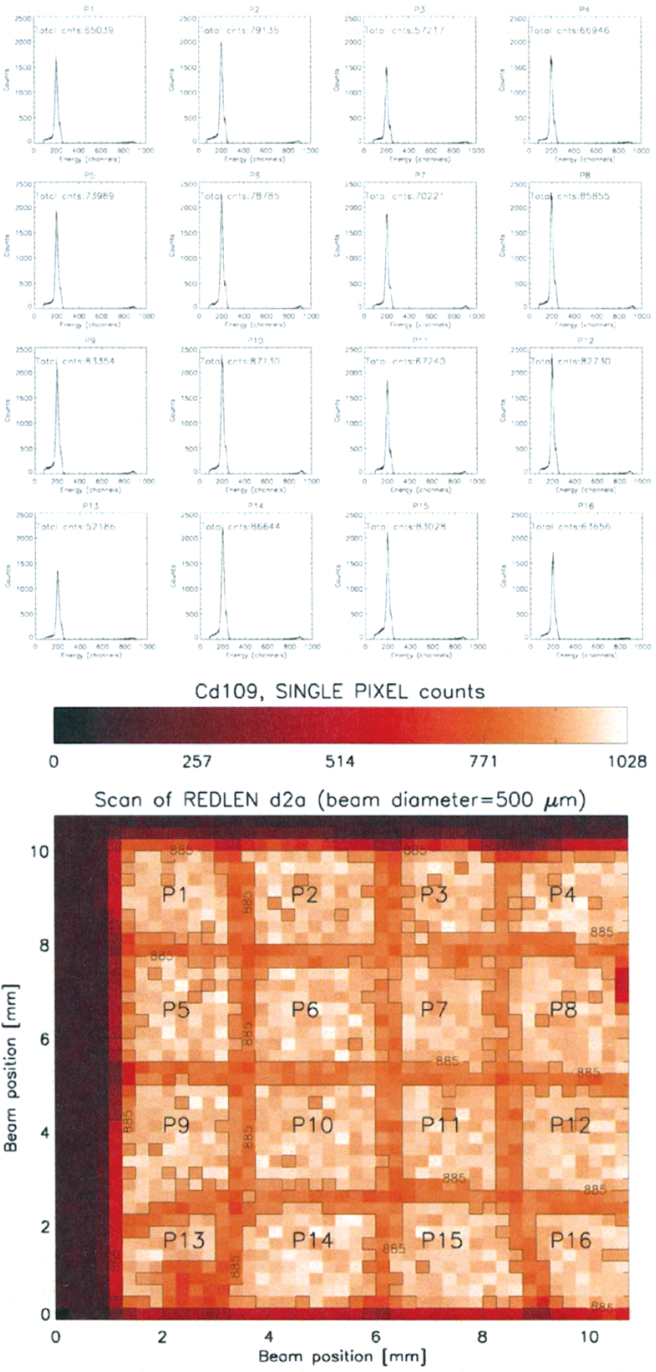

Fig. 6 The upper part displays the 16 pixel spectra obtained when illuminating detector $2 \mathrm{da}$ uniformly with ${ }^{109} \mathrm{Cd} \mathrm{X}$-rays. The ${ }^{109} \mathrm{Cd}$ scan was performed with a $0.5 \mathrm{~mm}$ beam and with $(\mathrm{x}, \mathrm{y})$ steps of $0.25 \mathrm{~mm}$. The results are shown in the lower part as a $2 \mathrm{D}$ count-image.

The investigations were performed using two radioactive sources: a ${ }^{109} \mathrm{Cd}$ source for low energy scans $(22.1 \mathrm{keV}$ and 25 
$\mathrm{keV})$ and a ${ }^{57} \mathrm{Co}$ source for high energy scans (122 keV and $136 \mathrm{keV})$.

All measurements were performed with the common planar cathode biased at $-500 \mathrm{~V}$ and the pixel anodes at $0 \mathrm{~V}$. The pixel detector was illuminated from the side of the common planar electrode.

The upper part of Fig 6 displays the 16 pixel spectra obtained when illuminating detector $2 \mathrm{da}$ uniformly with ${ }^{109} \mathrm{Cd}$ $\mathrm{X}$-rays. The data analysis s/w was operated such that only socalled single pixel events are displayed. In other words, shared events for which two or more pixel were triggered by the same event are rejected. The spectra clearly show pixel-to-pixel count rate variations also given by the total counts in top of each spectrum. Remark the difference of almost $25 \%$ between the counts for pixel 10 and pixel 11. These pixels are both central pixels and are not influenced by edge effect.

The ${ }^{109} \mathrm{Cd}$ scan was performed with a $0.5 \mathrm{~mm}$ beam and with $(x, y)$ steps of $0.25 \mathrm{~mm}$. The results are shown in the lower part of Fig. 6 as a 2D count-image. Again only single pixel events are displayed which has the consequence that the count rate drops when the beam passes over the boarders between pixels where charge sharing is present. The pixel boundaries are therefore clearly visible in the map as given by the dark boarder lines with fewer counts. The dark feature seen for pixel 13 is an exception since it is caused by the $\mathrm{Ag}$ filled conducting glue which is used for the contact to the common planar electrode. However, the important thing to remark is that sizes of the pixels are varying and that the pixel size is strongly correlated with the pixel counts in the upper plots, e.g. compare pixel 10 and pixel 11. It should also be remarked that the count rate variations within the pixels are very small and that the count rate inside the pixel is constant without pixel-to-pixel variations. We can conclude that the pixel size variations seen in the lower part of Fig. 6 fully explains the pixel-to-pixel count rate variations for uniform illumination given in the upper part of Fig. 6 The present measurements do also not show in-efficiencies and charge collection problems as those reported by [4] for a number of pixelated CZT detectors.

The X-ray photons of the ${ }^{109} \mathrm{Cd}$ source are stopped within $0.1 \mathrm{~mm}$ below the common planar electrode surface. In order to investigate whether the effective area variations are surface correlated we repeated the measurements with a ${ }^{57} \mathrm{Co}$ photon beam (122 and $136 \mathrm{keV}$ ) which has a mean free path of $\sim 1.7$ $\mathrm{mm}$ inside the detector CZT volume.

The results are displayed in Fig. 7. The upper plots show the 16 pixels spectra obtained by uniformly illumination of the detector with photons from the ${ }^{57} \mathrm{Co}$ source. The total number of detected counts are given in the top of each pixel plot. The pixel to pixel count variation is very similar to the one found when illuminating the detector with ${ }^{109} \mathrm{Cd} \mathrm{X}$-rays. The count difference between pixel 10 and pixel 11 is again $\sim 25 \%$.

The lower part image of Fig. 7 displays the results of the ${ }^{57} \mathrm{Co}$ scans. In this case we could operate with a somewhat finer beam of $0.3 \mathrm{~mm}$ diameter; but again with steps $(\mathrm{x}, \mathrm{y})$ of $0.25 \mathrm{~mm}$. The image displays the number of counts of single pixel events as explained for Fig. 6. The pixel boarders are clearly visible and the effective pixel sizes are almost identical to those measured with the ${ }^{109} \mathrm{Cd}$ source

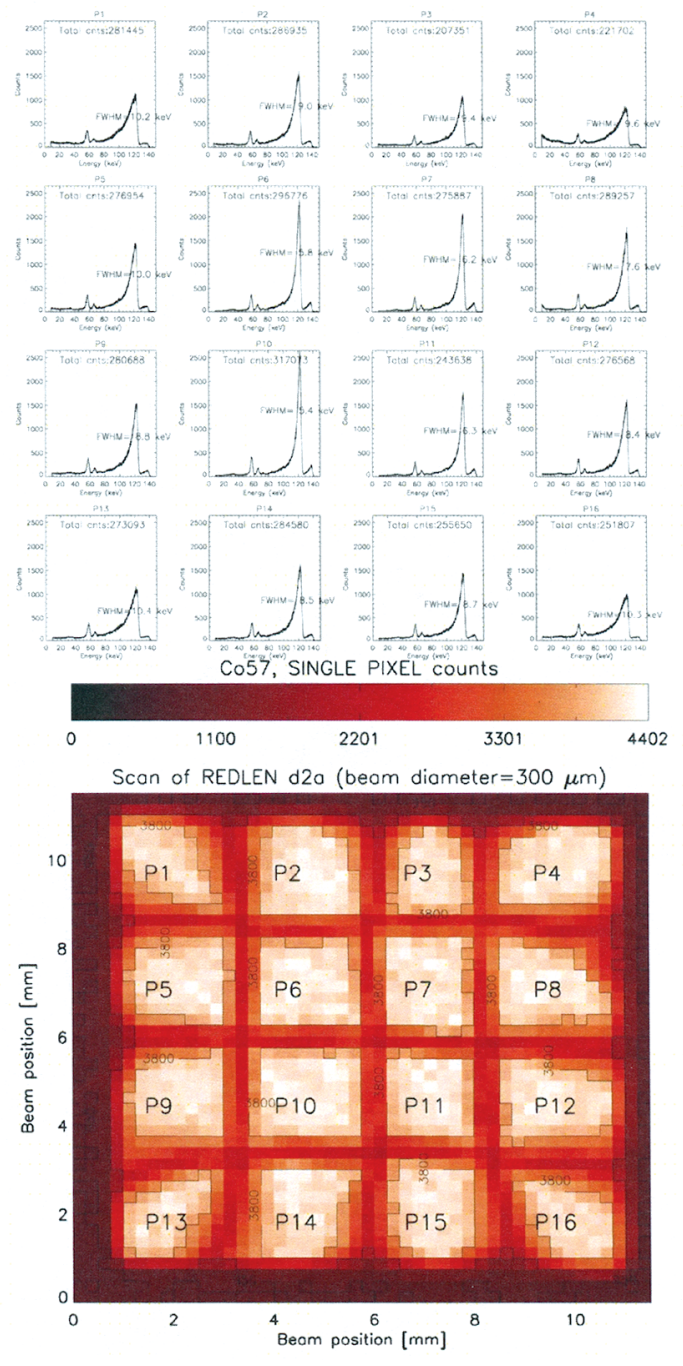

Fig. 7 The upper part displays the 16 pixel spectra obtained when illuminating detector 2 da uniformly with photons form the ${ }^{57} \mathrm{Co}$ source. The scan was performed with a $0.3 \mathrm{~mm}$ beam and with $(x, y)$ steps of $0.25 \mathrm{~mm}$. The results are shown in the lower part as a $2 \mathrm{D}$ count-image.

The preliminary conclusion is therefore that the effective pixel shape(size) does not depend on the photon interaction depth. This is surprising since we believe that the shape variations are caused by non-uniform electric fields inside the detector volume. Such fields can be formed by crystal defects and cause image distortions as demonstrated in [5], [6]. From the present results, it cannot be excluded that field distortions close to the pixel electrodes are responsible for the image distortions. Issues associated with attachment/assembly (delamination, smearing of low temperature solder, 
misalignment) could also possibly contribute to the scanned pixel size variation as well. In order to clarify this we plan to perform a full 3D analysis employing the DOI method [2], [3] to determine also the depth coordinate.

\section{CONCLUSION}

We have conducted pixel to pixel uniformity measurements on Redlen CZT detectors. Result for full illumination and scan consistently shown large count rate variations between pixels. We believe that the pixel-to-pixel non-uniformity is related to the non-uniform internal electric field profile. It is at present unclear what is disturbing the internal electric field. Other groups [5], [6] have found similar effects for CZT pixel detectors. The explanation in [5], [6] relates to extended defects and residual strains existing inside the crystal.

We plan to perform a full 3D analysis employing the DOI method on the CZT detector to reveal the electron drift path close to the pixels function of beam position.

The discussed pixel-to-pixel non-uniformities of the Redlen CZT pixel detectors are not a serious concern for the ASIM MXGS instrument. MSGS imaging will be performed with a coded mask with $10 \mathrm{~mm} \times 10 \mathrm{~mm}$ holes such that the variations will be smoothed over 16 detector pixels.

\section{ACKNOWLEDGMENT}

We thank Redlen Technology for their support and discussion.

\section{REFERENCES}

[1] Kuvvetli, I.; Budtz-Jørgensen, C., "Measurements of charge sharing effects in pixelated CZT/CdTe detectors," Nuclear Science Symposium Conference Record, 2007. NSS '07. IEEE Volume 3, Oct. 26 2007-Nov. 32007 Page(s):2252 - 2257

[2] M. A. J. van Pamelen and C. Budtz-Jørgensen, "Novel electrode geometry to improve the performance of CdZnTe detectors," Nucl. Instr. \& Methods Phys. Res. A, vol. A403, pp. 390-398, 1997.

[3] M. A. J. van Pamelen and C. Budtz-Jørgensen, "CdZnTe drift detector with correction for hole trapping," Nucl. Instr. \& Methods Phys. Res. A, vol. A411, pp. 197-200, 1998.

[4] M. D. Wilson, P. Seller, J. Marchal, Z.-J. Xin, C. Hansson, R. Cernik, M. C. Veale, V. Perumal, "X-Ray Performance of Pixelated CdZnTe Detectors", R12-24 in this conference.

[5] G. S. Camarda, A. E. Bolotnikov, G. Yang, Y. Cui, A. Hossain, R. B. James, "Effects of Extended Defects on the Internal Electric Field and Charge Trapping in CdZnTe Detectors", R02-3 in this conference.

[6] A. E. Bolotnikov, S. Camarda, Y. Cui, A. Hossain, G. Yang, H. W. Yao, and R. B. James, "Internal electric-field-lines distribution in $\mathrm{CdZnTe}$ detectors measured using X-ray mapping", SORMA 2008, submitted to TNS IEEE, accepted for publication. 